\title{
Regadenoson stress in patients with asthma and COPD: A breath of fresh air
}

\author{
Randall C Thompson, MD, FASNC
}

See related article, pp. 681-692

An important practical issue in contemporary nuclear cardiology laboratories regards the method of stress for patients who have asthma or chronic obstructive pulmonary disease (COPD). These patients are frequently not able to exercise adequately for testing, and the two most commonly used pharmacologic stress agents are at least relatively contraindicated with this condition. Both intravenous adenosine and dipyridamole non-specifically activate adenosine receptors, including the $\mathrm{A} 2 \mathrm{~b}$ receptor, which can lead to bronchospasm (increased pulmonary resistance and decreased pulmonary compliance) by stimulating the release of bronchoconstrictor mediators. ${ }^{1}$ Most experienced nuclear cardiologists can recall at least one case of severe respiratory distress requiring rescuscitation (or worse) induced by one of these agents. The risk of potentially severe bronchospastic reactions is greater in patients with a history of asthma, and these agents should therefore generally be avoided in this group. Bronchospasm is listed as an absolute contraindication in the package insert for adenosine, and ASNC guidelines for stress agents likewise caution that asthmatic patients with wheezing should not undergo testing with adenosine or dipyridamole. ${ }^{2}$ Unfortunately, dobutamine, the commonly used alternative pharmacologic stress agent which is considered to be safe for these patients, is less effective at augmenting coronary blood flow and is therefore considered to be an inferior test agent. Dobutamine is also unpopular among patients and laboratory staff because of a high incidence of side effects, the need to stop beta-blockers completely, and the lengthy time of the exam protocol.

From the Department of Cardiology, St. Luke's Mid America Heart Institute, Kansas City, MO.

Reprint requests: Randall C Thompson, MD, FASNC, Department of Cardiology, St. Luke's Mid America Heart Institute, Kansas City, MO; rthompson@saint-lukes.org.

J Nucl Cardiol 2012;19:647-8.

$1071-3581 / \$ 34.00$

Copyright $(\subset 2012$ American Society of Nuclear Cardiology.

doi:10.1007/s12350-012-9565-2
Regadenoson and other selective A2a adenosine receptor agonists are designed to have much less activity at the $\mathrm{A} 2 \mathrm{~b}$ receptor site, and thus, should theoretically be less likely to induce bronchospasm than adenosine or dipyridamole. Two small studies reported in 2008 provided some confirmation of the safety of regadenoson in patients with asthma and COPD. ${ }^{3,4}$ In the same issue of the Journal, Prenner et $\mathrm{al}^{5}$ report on a relatively large study of the effects of regadenoson on pulmonary function in patients with asthma and COPD. A total of 999 patients with coronary disease or risk factors were randomized to receive regadenoson or placebo in a 2:1 fashion with subsequent serial pulmonary function tests and clinical assessment. The results are quite reassuring that regadenoson is unlikely to induce significant bronchospasm in patients similar to the ones studied. Not only was the primary endpoint, $>15 \%$ decrease in FEV1 from baseline to 2 hours after administration, not different between the treatment and placebo arms in either the asthma or COPD stratum, but there was also no signal to suggest bronchospastic risk in subset analysis or at any time point post administration. No severe pulmonary adverse reactions were reported, and only one regadenoson patient received aminophylline for bronchospasm. Also, the individual regadenoson and placebo subjects who had the most significant decreases in FEV1 and $\mathrm{O} 2$ saturation had similar levels of reduction from baseline. The authors present extensive details on the response of their subjects, and the pulmonary safety of regadenoson is reassuring in each analysis.

\section{THE NEED FOR CONTINUED CAUTION IN PATIENTS WITH COPD AND ASTHMA}

While the study by Prenner et al is well executed and an important contribution, it does not prove that regadenoson never induces bronchospasm. Thus, as the authors point out, continued caution is warranted in high-risk pulmonary patients. Very serious bronchospastic reactions to adenosine and dipyridamole are uncommon, and it is possible that the sample size of the Prenner study is simply not large enough to detect rare serious reactions from regadenoson. Older reports of these incidents include dipyridamole-induced severe bronchospasm in two of 800 patients in a study by 
Eagle and Boucher, and in six of 3,911 patients reported by Ranhosky et al. ${ }^{6,7}$ Adenosine was reported by Cerqueira et $\mathrm{al}^{8}$ to cause severe bronchospasm in six of 9,256 patients enrolled in a multicenter trial registry. In contrast, the study by Prenner exposed a smaller number of patients (672) to regadenoson, although all of them had asthma or COPD.

The study by Prenner also included only a small number of subjects with severe lung disease. For example, only two asthma patients were in the NHLBI step criteria that include oral corticosteroid treatment, and only six COPD patients who met GOLD criteria stage IV (very severe) were in the study. These patients are most at risk for respiratory reactions and the reassuring results regarding the pulmonary response of regadenoson should not necessarily be extrapolated to this group.

Importantly, and as pointed out by the authors, the study by Prenner et al was performed in patients who had stable asthma or COPD. Patients in whom recent changes were made in pulmonary medications were excluded from this trial. Physicians should continue to refrain from using regadenoson in patients with unstable pulmonary conditions.

The investigators involved in the study reported by Prenner should be congratulated on their careful investigation and this important contribution. For patients with asthma or COPD requiring pharmacologic stress myocardial perfusion imaging and who are similar to the subjects who were included in the trial, a selective A2a adenosine agonist such as regadenoson would logically be preferable than using adenosine or dipyridamole. For patients with more advanced asthma or COPD and for those whose lung condition is less than stable, other strategies should still be employed.

\section{References}

1. Polosa R, Rorke S, Holgate ST. Evolving concepts on the value of adenosine hyper-responsiveness in asthma and chronic obstructive pulmonary disease. Thorax 2002;57:649-54.

2. Henzlova MJ, Cerqueira MD, Taillefer R, Mahmarian JJ, Yao SS. Quality Assurance Committee of the American Society of Nuclear Cardiology. Stress protocols and tracers. J Nucl Cardiol 2009. doi: 10.1007/s12350-009-9062-4.

3. Leaker BR, O'Connor B, Hansel TT, et al. Safety of regadenoson, an adenosine A2A receptor agonist for myocardial perfusion imaging, in mild asthma and moderate asthma patients: A randomized, double blind, placebo-controlled trial. J Nucl Cardiol 2008;15:329-36.

4. Thomas GS, Tammelin BR, Schiffman GL, et al. Safety of regadenoson, a selective adenosine $\mathrm{A} 2 \mathrm{~A}$ agonist, in patients with chronic obstructive pulmonary disease: A randomized, doubleblind, placebocontrolled trial (RegCOPD trial). J Nucl Cardiol 2008; $15: 319-28$

5. Prenner BM, Bukofzer S, Behm S, Feaheny K, McNutt BE. A randomized, double-blind, placebo-controlled study assessing the safety and tolerability of regadenoson in subjects with asthma or chronic obstructive pulmonary disease. J Nucl Cardiol 2012. doi: 10.1007/s12350-012-9547-4.

6. Eagle KA, Boucher CA. Intravenous dipyridamole infusion causes severe bronchospasm in asthmatic patients. Chest 1989;95:258-9.

7. Ranhosky A, Kempthorne-Rawson J, the Intravenous Dipyridamole Thallium Imaging Study Group. The safety of intravenous dipyridamole thallium myocardial perfusion imaging. Circulation 1990;81:1205-9.

8. Cerqueira MD, Verani MS, Schwaiger M, Heo J, Iskandrian AS. Safety profile of adenosine stress perfusion imaging: Results from the Adenoscan Multicenter Registry. J Am Coll Cardiol 1994;23:384-9. 